\title{
Generating Realistic Optical Transport Network Topologies
}

\author{
Claunir Pavan, Rui Manuel Morais, José R. Ferreira da Rocha, and Armando Nolasco Pinto
}

\begin{abstract}
We address the problem of generating physical realistic optical transport network topologies. This type of network has characteristics that differ from scale-free networks, such as the Internet. Based on the analysis of a set of real transport topologies, we identify and assess relevant characteristics. A method to generate realistic topologies is proposed. The proposed method is validated by comparing the characteristics of computer-generated and real-world optical transport networks.
\end{abstract}

Index Terms-Network topology design; Physical topology; Network survivability; Transport networks.

\section{INTRODUCTION}

C omputer-generated (CG) network topologies are often employed to perform simulations and analysis of algorithms in telecommunications networks. The reason for using CG topologies is due to the lack of available real-world networks in a large number for extensive studies [1]. Usually, completely random topologies do not have the required characteristics [2]. Therefore, their use can lead to incorrect decision making, such as underestimation of the impact of failures in a network. Thus, it is crucial to have a method to generate network topologies that resemble real-world transport networks.

Network topology generators [3-11] are extensively available in the literature. In [3] the author presents a model for generating random graphs in which the nodes are distributed over a plane, and links are added to the graph using a probability function based on the Euclidean distance between the nodes. In $[4,5]$ the multilevel hierarchy found in the Internet is used

Manuscript received September 21, 2009; revised November 23, 2009; accepted November 27, 2009; published December 24, 2009 (Doc. ID 117402).

C. Pavan (e-mail: pavan@ua.pt), J. R. Ferreira da Rocha (e-mail: frocha@ua.pt), and A. N. Pinto (e-mail: anp@ua.pt) are with the Departamento de Electrónica, Telecomunicações e Informática, Universidade de Aveiro and Instituto de Telecomunicações, 3810193, Aveiro, Portugal.

R. M. Morais (e-mail: rmorais@av.it.pt) is with the Instituto de Telecomunicações, 3810-193, Aveiro, Portugal.

Digital Object Identifier 10.1364/JOCN.2.000080 to generate Internet-like topologies. In [6] the authors extract the autonomous system and router level topologies from the Internet, and from that realistic core, topologies are generated. In [7] the authors show that the nodal degree distribution of the autonomous system level topologies follow a power law. From that, several topology generators have been built based on power laws [8-11].

However, previous efforts have been focused on topologies resembling the Internet, which is a scale-free network [12]. Scale-free networks contain a few nodes with a very high number of links, while most nodes have just a few links. The nodal degree distribution tends to follow a power law [12].

In this paper, we are concerned with optical transport networks with survivable topologies. The characteristics of this kind of network differs from scale-free networks. For instance, it is extremely rare to find nodes that have significantly more or fewer links than the average. Thus, topologies that resemble the Internet or topologies based on power laws are not suitable for optical transport network analysis.

The starting point of our work is an extensive analysis of real-world transport networks to identify their relevant characteristics. Next a model is proposed to generate topologies that resemble optical transport networks.

The paper is organized as follows: In Section II, we present a set of real-world optical transport networks and analyze their main characteristics. In Section III, we develop a method for generating topologies with these characteristics. The validation of the method is provided in Section IV. In Section V, the main conclusions of this paper are summarized.

\section{TRANSPORT NETWORK TOPOLOGY CHARACTERISTICS}

To identify and study the key variables of real transport networks, we have collected a set of 29 topologies of real survivable transport networks (all that we have found). The number of nodes ranges from 9 to 100 (see Table I). Next we analyze the characteristics of these network topologies, with the aim of 
TABLE I

REAL-WORLD REFERENCE NETWORKS

\begin{tabular}{|c|c|c|c|c|}
\hline Number & Network & $N$ & $L$ & $\langle\delta\rangle$ \\
\hline 1 & VIA Network [13] & 9 & 12 & 2.67 \\
\hline 2 & BREN [14] & 10 & 11 & 2.20 \\
\hline 3 & RNP [15] & 10 & 12 & 2.40 \\
\hline 4 & vBNS [16] & 12 & 17 & 2.83 \\
\hline 5 & CESNET [17] & 12 & 19 & 3.17 \\
\hline 6 & NSFNET [18] & 14 & 21 & 3.00 \\
\hline 7 & Italy [19] & 14 & 29 & 4.14 \\
\hline 8 & Austria [20] & 15 & 22 & 2.93 \\
\hline 9 & Mzima [21] & 15 & 19 & 2.53 \\
\hline 10 & ARNES [22] & 17 & 20 & 2.35 \\
\hline 11 & Germany [23] & 17 & 26 & 3.06 \\
\hline 12 & Spain [24] & 17 & 28 & 3.29 \\
\hline 13 & LambdaRail [25] & 19 & 23 & 2.42 \\
\hline 14 & Memorex [26] & 19 & 24 & 2.53 \\
\hline 15 & CANARIE [27] & 19 & 26 & 2.74 \\
\hline 16 & EON [28] & 19 & 37 & 3.89 \\
\hline 17 & ARPANET [29] & 20 & 32 & 3.20 \\
\hline 18 & PIONIER [30] & 21 & 25 & 2.38 \\
\hline 19 & Cox $[31]$ & 24 & 40 & 3.33 \\
\hline 20 & SANET [32] & 25 & 28 & 2.24 \\
\hline 21 & NEWNET [33] & 26 & 31 & 2.38 \\
\hline 22 & Portugal [34] & 26 & 36 & 2.77 \\
\hline 23 & RENATER [35] & 27 & 35 & 2.59 \\
\hline 24 & GEANT2 [36] & 32 & 52 & 3.25 \\
\hline 25 & LONI [37] & 33 & 37 & 2.24 \\
\hline 26 & Metrona [38] & 33 & 41 & 2.48 \\
\hline 27 & Omnicom [39] & 38 & 54 & 2.84 \\
\hline 28 & Internet $2[40]$ & 56 & 61 & 2.18 \\
\hline 29 & USA 100 [41] & 100 & 171 & 3.42 \\
\hline
\end{tabular}

identifying the relevant variables for the adequate characterization of transport networks.

In general, a real-world transport network topology can be seen as a graph over a two-dimensional plane. The nodes are distributed according to the expected traffic demand in each geographic area. Thereby, we often can identify regions with more nodes than the others. Here a region stands for a number of cities or countries (it depends on the geographic span). Figure 1 shows a possible set of regions on the European Optical Network (EON) topology. Although regions without any or very few nodes are pretty likely to be found, we can frequently find a set of nodes that form a cycle when the region holds a set of at least three nodes. Cycles of nodes allow survivability because each pair of nodes has two disjoint interconnecting paths. When a node is unique within a region, the survivability tends to be provided by connecting the node to at least two nodes of neighbor regions, forming a cycle; in the case of regions with two nodes, the nodes tend to be directly connected and each one tends to be directly connected to at least a single node in a neighbor region.

Besides this holistic view, we were able to identify a

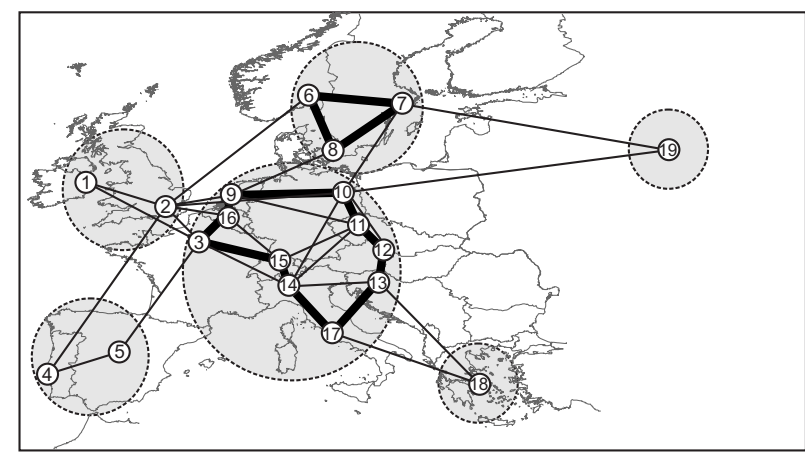

Fig. 1. Physical topology of the European Optical Network (EON). The nodes are interconnected with optical cables and distributed across a geographic area. Some regions are more densely populated with nodes and links than others. Regions with a cluster of nodes often present cycles (see the strong links).

few variables that characterize transport network topologies. The most relevant are the nodal degree, $\delta$; the number of hops, $h$; link-disjoint pairwise connectivity, $\omega$; node-disjoint pairwise connectivity, $\theta$; and clustering coefficient, $c$. In the following we present each variable in more detail and relate them to the set of real-world transport networks, shown in Table I.

To determine the distribution of $\delta, h, \omega, \theta$, and $c$, we performed a variety of goodness-of-fit nonparametric statistical tests. Using the one-sample KolmogorovSmirnov test [42], we verified that the nodal degree of 21 networks from Table I follows a Poisson distribution at the 0.05 significance level, and 4 networks (18, $21,23,27)$ follow a Poisson distribution at the 0.01 significance level. Figure 2 presents the nodal degree relative frequency distribution (gray bars) for the USA 100 network and the Poisson probability function, with $\lambda=\langle\delta\rangle=3.42$ (solid curve).

We have noticed that the networks failing the test at both significance levels (networks 17, 25, 26, 28) are quasi-regular; i.e., the nodal degree is almost the

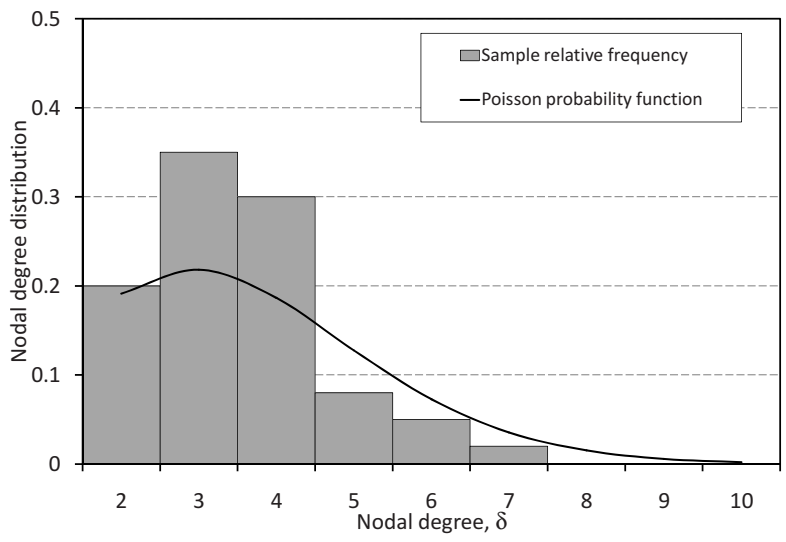

Fig. 2. Nodal degree relative frequency and the Poisson probability function, with $\lambda=\langle\delta\rangle=3.42$ for the USA 100 network. We verified that real transport networks tend to follow a Poisson distribution for the nodal degree. 
same for all nodes and the variance is small. These results confirm the results obtained in $[12,43]$. According to [43], the nodal degree of optical transport networks tends to follow a Poisson distribution. These networks are also called exponential networks because the probability that a node is connected to $k$ other nodes tends to decrease exponentially for larger $k$ [12].

In survivable topologies, the minimum nodal degree is required to be two, i.e., $\delta_{\min } \geqslant 2$. Note that this feature is necessary but not sufficient for survivability purposes. The nodal degree in our reference networks ranges from 2 to 10, and the average nodal degree ranges from $\langle\delta\rangle=2.18$ to $\langle\delta\rangle=4.14$ [see Fig. 3(a)]. Considering all networks, we have obtained $\langle\delta\rangle^{*}=2.8$. We use the asterisk index to indicate that the value of the parameter is obtained from the set of networks rather than a particular network. The standard deviation for nodal degree ranges from 0.4 to 2 .

Nodes are reachable through a single-hop or a multihop interconnection. The latter requires the crossing of intermediary nodes and links. The average number of hops, $\langle h\rangle$, is determined by the physical network topology and the routing algorithm. In this work we assume a shortest path routing. Assuming bidirectional
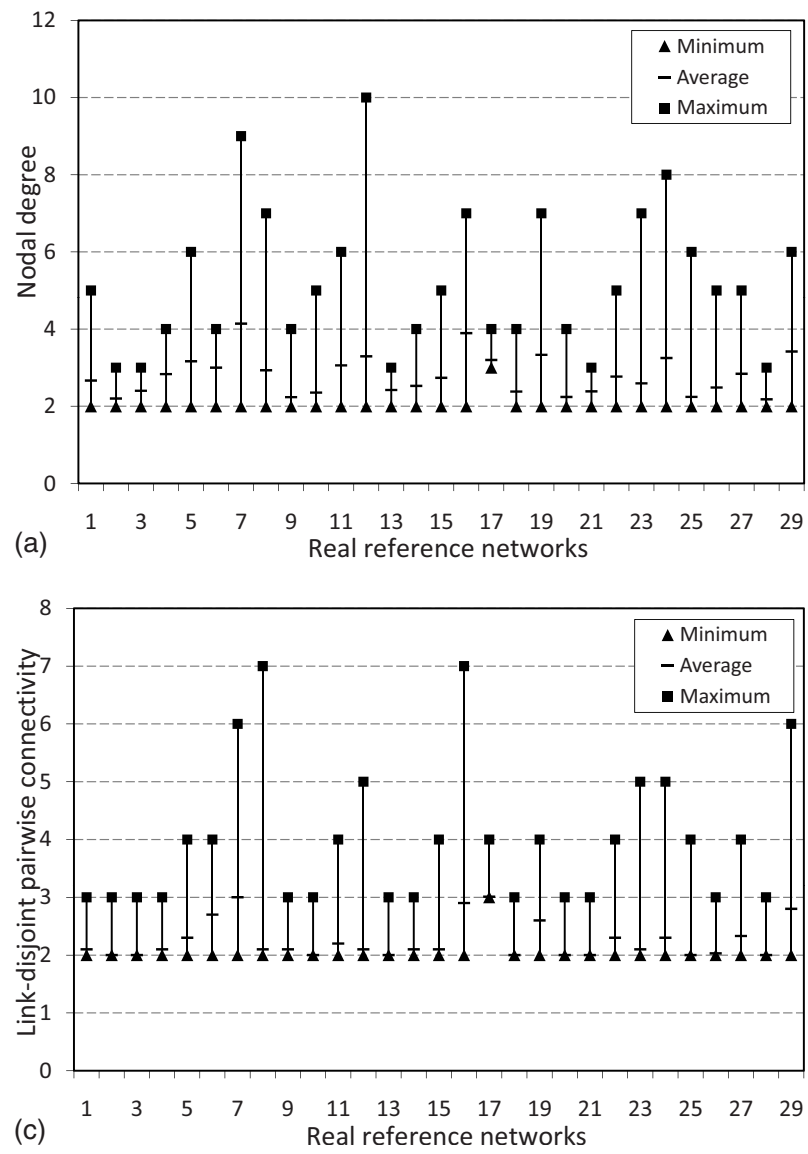

paths, the average number of hops, $\langle h\rangle$, is by definition the summation of the number of hops of all possible node pairs divided by the number of possible node pairs,

$$
\langle h\rangle=\frac{2}{N(N-1)} \sum_{i=1}^{N-1} \sum_{j=i+1}^{N} h_{i j},
$$

where $N$ is the total number of nodes and $h_{i j}$ is the number of hops between the nodes $i$ and $j$.

In Fig. 3(b) we can see that the number of hops varies between 1 and 21 . Regarding the average number of hops, $\langle h\rangle$, the values range from 2 to 8.5 and the standard deviation is in the range of 0.7 to 4.6. Considering all networks we have $\langle h\rangle^{*}=3.4$. We observed that larger networks are sparser, which tends to lead to a higher average number of hops, $\langle h\rangle$.

Since a failure may affect various shared resources, the connectivity must be sufficient to allow recovery techniques to be employed. Recovery techniques usually rely on node and/or link-disjoint paths to ensure that both the working and backup paths will not be affected by the same single failure [44]. Connectivity is a measure that depends on the number of disjoint paths. Link-disjoint pairwise connectivity, $\omega_{i j}$, is the
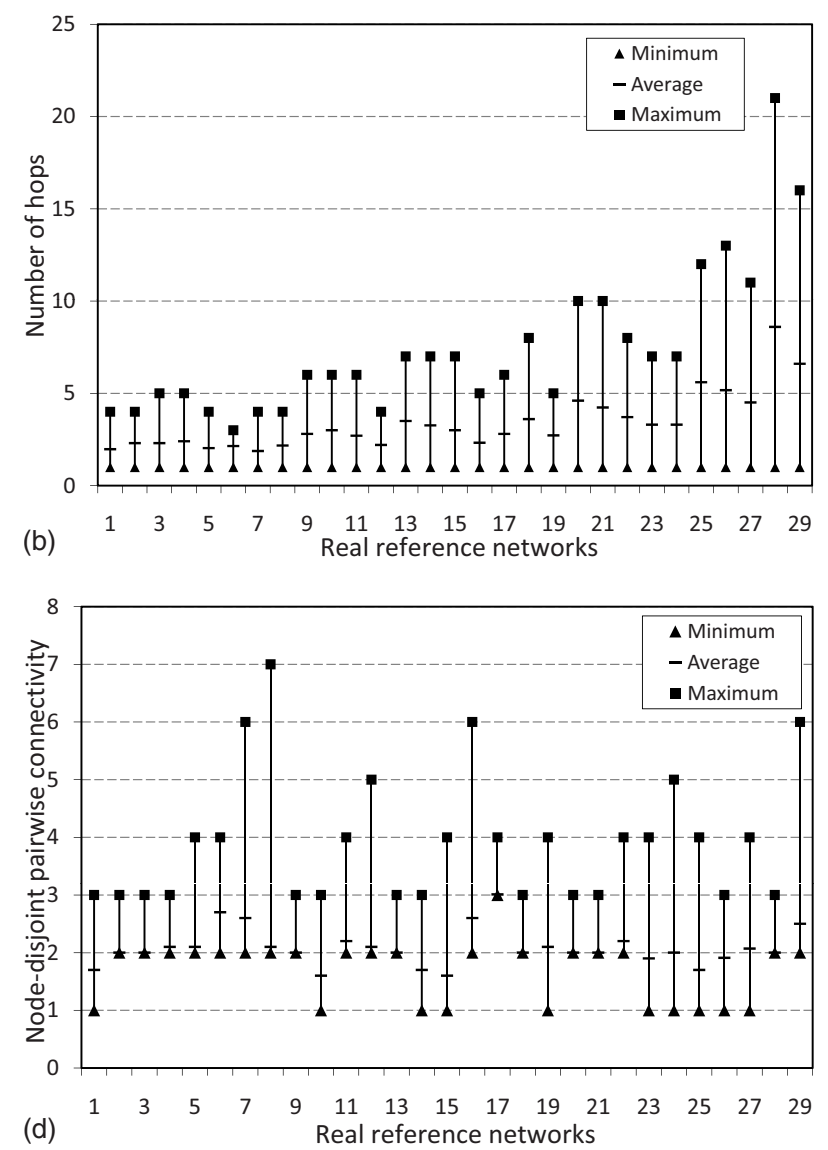

Fig. 3. The minimum, average, and maximum values of (a) the nodal degree, (b) the number of hops, (c) the link-disjoint pairwise connectivity, and (d) the node-disjoint pairwise connectivity for 29 real-world network topologies. 
number of link-disjoint paths between the node pair $i j$. That is, between the nodes $i$ and $j$ there are $\omega_{i j}$ paths in which the intermediary links are not shared. The value of $\omega_{i j}$ also indicates the allowed number of link failures. For instance, a network topology with $\omega_{i j}=2$ for all pairs of nodes tolerates single link failures $[4,45]$. For $\omega_{i j}=3$ at most two link failures are tolerated and so forth. Adding the link-disjoint pairwise connectivity of all node pairs and dividing by the number of possible bidirectional node pairs, we obtain the average link-disjoint pairwise connectivity, $\langle\Omega\rangle$, for a network,

$$
\langle\Omega\rangle=\frac{2}{N(N-1)} \sum_{i=1}^{N-1} \sum_{j=i+1}^{N} \omega_{i j} .
$$

Referring to Fig. 3(c) we can see that survivable networks have at least two link-disjoint paths between each pair of nodes, $\omega_{i j} \geqslant 2$. Furthermore, this value goes up to seven in our sample, $\omega_{i j} \leqslant 7$. The standard deviation obtained is in the range of 0 to 0.9 . We have noticed that the average link-disjoint pairwise connectivity, $\langle\Omega\rangle$, increases with $\langle\delta\rangle$. Considering all networks, we have $\langle\Omega\rangle^{*}=2.25$.

Two paths are node-disjoint if they have no nodes in common other than the source and destination. Nodedisjoint pairwise connectivity, $\theta_{i j}$, is the number of node-disjoint paths between the node pair $i j$. The value of $\theta_{i j}$ also indicates the tolerance to node failures. Since a node-disjoint path also implies a linkdisjoint path, a network with $\theta_{i j} \geqslant 2$ for all node pairs allows survivability against both node and link failures [46]. We can obtain the average node-disjoint pairwise connectivity, $\langle\Theta\rangle$, for a network with

$$
\langle\Theta\rangle=\frac{2}{N(N-1)} \sum_{i=1}^{N-1} \sum_{j=i+1}^{N} \theta_{i j} .
$$

In terms of node-disjoint pairwise connectivity [Fig. $3(\mathrm{~d})$ ], we have noticed that $\langle\Theta\rangle$ tends to increase with $\langle\delta\rangle$. Some of our real-world reference topologies do not tolerate node failures; see the networks in which the minimum node-disjoint pairwise connectivity is 1 . For our reference networks, the values of node-disjoint pairwise connectivity satisfies $1 \leqslant \theta_{i j} \leqslant 7$, with standard deviation in the range of 0 to 1 . The average node-disjoint pairwise connectivity, $\langle\Theta\rangle$, for survivable topologies against single node failures ranges between 2 and 3. Considering all networks we have $\langle\Theta\rangle^{*}=2.21$.

The clustering coefficient of a node, $c_{i}$, quantifies how close its neighbors are to being a full mesh. The neighborhood of a node, $n_{i}$, is the set of nodes that are directly connected to the node $i$. The value of $c_{i}$ varies from 0 to 1 , being 1 if the neighborhood forms a full mesh and 0 if none of the neighbors are directly con- nected [47]. For undirected and connected graphs, the clustering coefficient of a node [47] is defined as

$$
c_{i}=\frac{2 t_{i}}{\delta_{i}\left(\delta_{i}-1\right)},
$$

where $t_{i}$ is the number of triangles that exists involving the node $i$ and its neighbors, $n_{i}$, and $\delta_{i}$ is the nodal degree of the node $i$. The clustering coefficient of the network, $\langle c\rangle$, is the average clustering coefficient of all nodes in the network,

$$
\langle c\rangle=\frac{1}{N} \sum_{i=1}^{N} c_{i}
$$

The clustering coefficient of the nodes in our real networks ranges from 0 to 1 . The clustering coefficient of the network ranges from 0 to 0.69 , with standard deviation in the range of 0 and 0.4. Considering all networks, we have obtained $\langle c\rangle^{*}=0.19$.

\section{PROPOSED METHOD}

The proposed method is based on the Waxman model [3]. This choice was made because topologies generated with the Waxman model tend to follow a Poisson distribution for the nodal degree $[48,49]$ (the same trend of real-world optical transport networks). To more accurately satisfy survivable transport network characteristics, our method differs from the Waxman model in the following ways: (a) the plane is divided into regions and (b) node placement and connectivity obey certain constraints.

In the Waxman approach, the probability of a pair of nodes being directly connected is

$$
P(i, j)=\beta \exp \frac{-d(i, j)}{\zeta \alpha},
$$

where $d(i, j)$ is the Euclidean distance between the nodes $i$ and $j, \zeta$ is the maximum distance between any two nodes, and $\alpha$ and $\beta$ are parameters in the range of $(0,1]$. Increasing the value of $\alpha$ leads to a larger ratio of long links to short links, whereas the probability of links between any pair of nodes increases with $\beta$. Figure 4 shows a network topology generated with the Waxman model. As can be seen in Fig. 4, the Waxman model produces topologies with nodes of degree 1 . Also, it does not guarantee a connected topology $[4,5]$. Moreover, as the Waxman model distributes the nodes randomly over the whole plane, links crossing the whole plane tend to appear. Therefore, the networks generated do not have the connectivity characteristics of real-world survivable transport networks.

Our proposed method models a survivable transport network as a set of interconnected smaller subnetworks and introduces constraints to guarantee the 


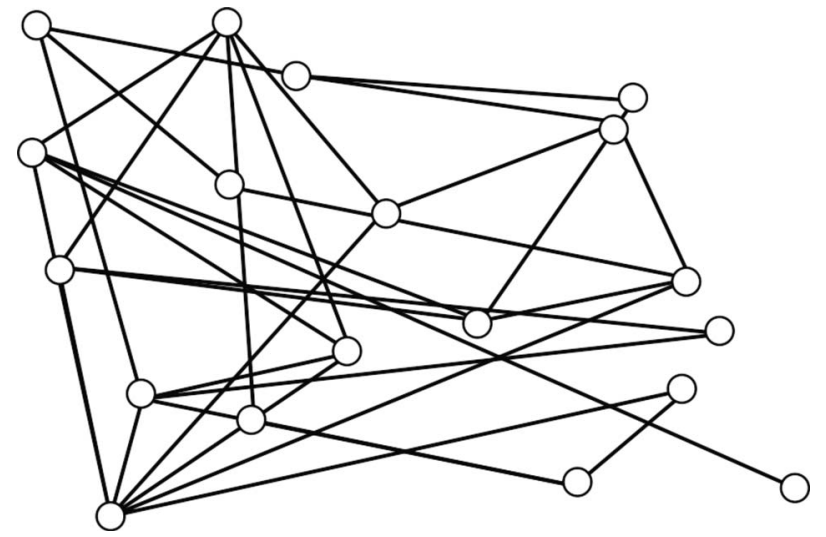

Fig. 4. A network generated by the Waxman model with $\alpha=0.4$ and $\beta=0.4$.

characteristics showed in the previous section. As presented in Table II, our method requires a set of nine inputs: the number of nodes, $N$; minimum and maximum mean nodal degrees, $\langle\delta\rangle_{\min }$ and $\langle\delta\rangle_{\max }$, used to specify the minimum and maximum number of links; the area of the plane $A$ (the plane is assumed to be a square with side $X=\sqrt{A}$ ); the number of regions, $R$, used as part of the strategy to resemble the connectivity of transport networks; and the minimum distance between the nodes, $d$, which restricts the distance between the nodes. The $\alpha$ and $\beta$ are parameters of the embedded Waxman link probability [3]. The number of simulations is specified by $\varphi$.

The method consists of the following steps:

- Divide the plane into $R$ regions.

- Assign and place the $N$ nodes into the regions.

- Interconnect the nodes inside each region.

- Interconnect the nodes between different regions.

- Add links to satisfy the mean nodal degree criterion.

The plane is partitioned into $R$ equal area regions. Each region has an area of

$$
A_{r}=\Delta X_{r}^{x} \Delta X_{r}^{y},
$$

where $\Delta X_{r}^{x}$ and $\Delta X_{r}^{y}$ are the dimensions of the region $r$. The number of horizontal and vertical divisions on the

TABLE II

InPut Variables for the Proposed Method

\begin{tabular}{cl}
\hline Variable & \multicolumn{1}{c}{ Description } \\
\hline$N$ & Number of nodes \\
$\langle\delta\rangle_{\min }$ & Minimum average nodal degree \\
$\langle\delta\rangle_{\max }$ & Maximum average nodal degree \\
$A$ & Area of the plane in arbitrary units \\
$R$ & Number of regions \\
$d$ & Minimum distance between two nodes \\
$\alpha$ & Waxman link probability parameter \\
$\beta$ & Waxman link probability parameter \\
$\varphi$ & number of simulations \\
\hline
\end{tabular}

plane depends on the given $R$, which is first decomposed into two numbers $(p 1, p 2)$. The number $p 1$ is the largest prime number such that $R$ is divisible by $p 1$. The number $p 2$ is the ratio between $R$ and $p 1$. Then $\Delta X_{r}^{x}=X / p 2$ and $\Delta X_{r}^{y}=X / p 1$. Thus, the plane is divided into $p 1$ rows and $p 2$ columns; see Fig. 5 in which $R=6$. In case of $R$ being a prime number, 1 is added to $R$ to allow this plane division strategy. The extra region remains without nodes.

Given the area of a region, $A_{r}$, and the minimum distance between two nodes, $d$, the maximum number of nodes that can be placed into a region is roughly

$$
n_{\max }=\frac{A_{r}}{d^{2}}
$$

To distribute the nodes the regions are chosen at random and the nodes are assigned to them, obeying the limit $n_{\max }$. Thereafter, the nodes are randomly placed over the respective regions, obeying the given minimum distance between the nodes, $d$ (which represents a blocked area around the nodes). In Fig. 5(b), the placed nodes are shown as black squares, whereas the gray squares represent the blocked area.

After the above procedure, we may have regions without nodes, with one, two, or more than two nodes. If a region has two or more nodes, an additional procedure is required, that is, if there are two nodes, they are directly connected; if there are more than two nodes, they are connected as a cycle. For regions with more than three nodes, the way the nodes are directly connected follows the Waxman link probability [3]. See a scenario for this phase in Fig. 5(b).

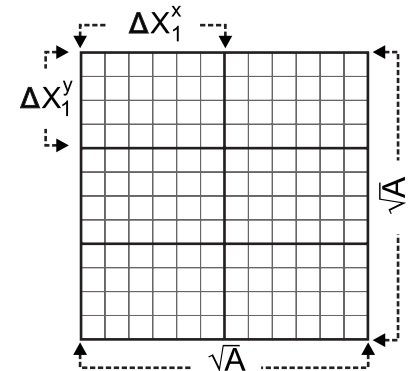

(a)

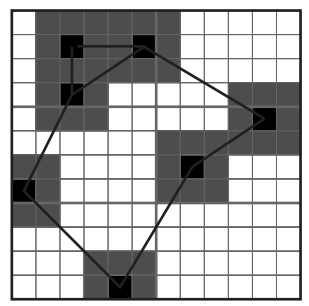

(c)

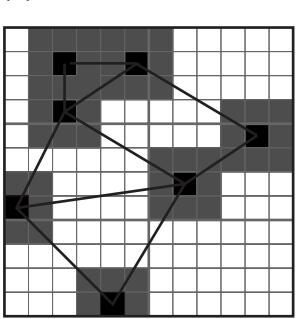

(d)

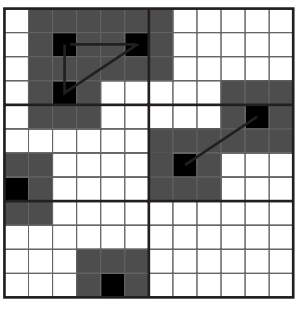

(b)

Fig. 5. (a) The plane and regions. (b) Node placing, connection, and blocked areas. (c) Region interconnection. (d) A possible network topology over a six-region plane. 
Once the nodes inside each region are interconnected, new links should be added to interconnect the regions. This process also follows the Waxman link probability; however, each node of the selected pair belongs to different regions. To guarantee that the generated topology will be survivable, some precautions should be taken: If a region has only one node, this node must be connected to at least two nodes of neighbor regions; if the region has only two nodes, each one must be connected to a node in neighbor regions; if a region has more than two nodes, at least two nodes must be connected to nodes of neighbor regions. Two nodes of a region can be connected to the same destination node in a neighbor region only if node-disjoint paths are not required.

At this phase we have a connected and survivable network topology (at least against single link failures). However, new links should be added until the given minimum mean nodal degree, $\langle\delta\rangle_{\min }$, is reached. This procedure is done following the Waxman link probability. Afterwards, a new topology is stored for each new link between $\langle\delta\rangle_{\min }$ and $\langle\delta\rangle_{\max }$. This procedure will generate several network topologies with the same node distribution, but with different average nodal degrees, $\langle\delta\rangle$. If the number of simulations, $\varphi$, is more than 1 , the nodes and links should be cleared from the plane and all procedures for the node distribution are done again with the same inputs.

The number of topologies that are generated during the algorithm run, $T$, depends on $\langle\delta\rangle_{\min },\langle\delta\rangle_{\max }, \varphi$, and $N$, and it is given by

$$
T=\varphi\left\lfloor\left(\frac{\langle\delta\rangle_{\max } N-\langle\delta\rangle_{\min } N}{2}\right)+1\right\rfloor .
$$

Given the minimum average nodal degree, $\langle\delta\rangle_{\text {min }}$; maximum average nodal degree, $\langle\delta\rangle_{\max }$; and total number of nodes, $N$, we achieve the number of different topologies that are generated during the algorithm run. The expression $\left(\langle\delta\rangle_{\max } N-\langle\delta\rangle_{\min } N\right) / 2$ gives the number of bidirectional links that remains to be included in an initial topology with $\langle\delta\rangle_{\min }$, until $\langle\delta\rangle$ $=\langle\delta\rangle_{\max }$. The $\varphi$ is the number of algorithm runs. The floor function is applied because it is not always possible to obtain topologies with the given $\langle\delta\rangle_{\min }$ and $\langle\delta\rangle_{\max }$, with $N$ nodes. Figure 5(d) shows an example of a network topology, and Fig. 6 shows the flow chart of the algorithm.

\section{EXPERIMENTS AND RESULTS}

To validate the proposed method, we have implemented a program following the flow chart in Fig. 6 . Before starting the generation of topologies we have to calibrate the generator. This means we need to find out adequate values for the input parameters.

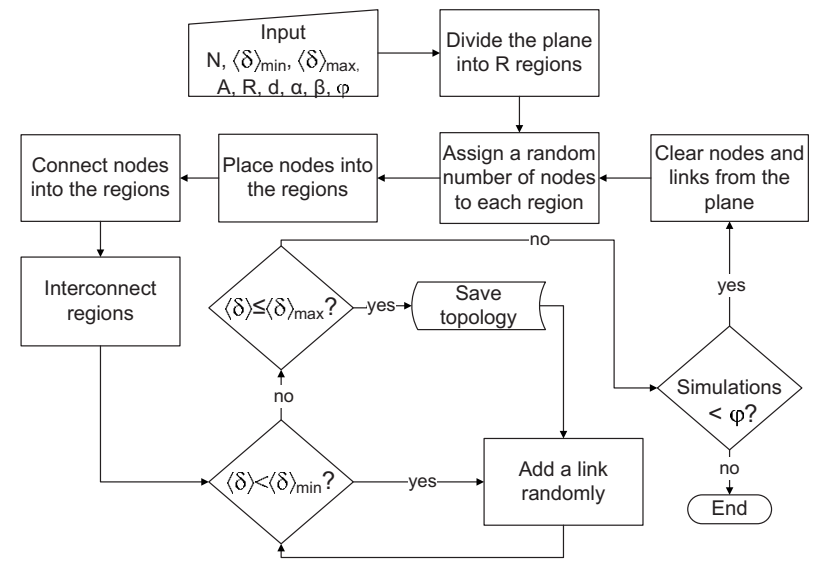

Fig. 6. A plane is divided into $R$ regions. A random number of nodes is assigned and placed in each region. After the nodes are interconnected, new links are added while the mean nodal degree is between $\langle\delta\rangle_{\min }$ and $\langle\delta\rangle_{\max }$.

For transport network topologies, the number of nodes $N$ varies from 10 to 100 nodes. The mean nodal degree is $2 \leqslant\langle\delta\rangle \leqslant 4$ as can be seen in Fig. 3(a). The area of the plane, $A$, must be large enough to accommodate $N$ nodes. Using the number of nodes, $N$, and the minimum distance between the nodes, $d$, the value of $A$ must be larger than $N d^{2}$. The number of regions, $R$, depends on the size of the plane and the number of nodes. A plane with more regions leads to more cycles and higher $\langle h\rangle$. We noticed that for transport networks a suitable range of values is between $4 \leqslant R \leqslant 20$.

For the experiments conducted in this work we have assumed the same $N$ and $\langle\delta\rangle$ of the real-world reference networks. The plane was assumed to be $A$ $=100^{2}$. The plane was partitioned into 12 regions, $R$ $=12$, and the minimum distance between the nodes was considered to be $2, d=2$. In [3] the author used $\alpha=0.4$ and $\beta=0.4$. To verify whether these values are appropriate for our method, we used the one-sample Kolmogorov-Smirnov test to obtain the best-fit curve to the link length distribution over all real-world topologies. Figure 7 shows that the curve fits well with the same values originally used by Waxman in [3], with mean error less than $2 \%$. Therefore we use $\alpha$ $=0.4$ and $\beta=0.4$.

The minimum, average, and maximum values for the nodal degree, $\delta$; number of hops, $h$; link-disjoint pairwise connectivity, $\omega$; and node-disjoint pairwise connectivity, $\theta$, for the generated networks have been calculated and are graphically represented in Fig. 8.

To identify whether the variables of computergenerated topologies follow the same distribution of the real-world ones, we have used the twoindependent-sample Kolmogorov-Smirnov test [42].

Regarding nodal degree, the tests have revealed that all computer-generated topologies follow the 


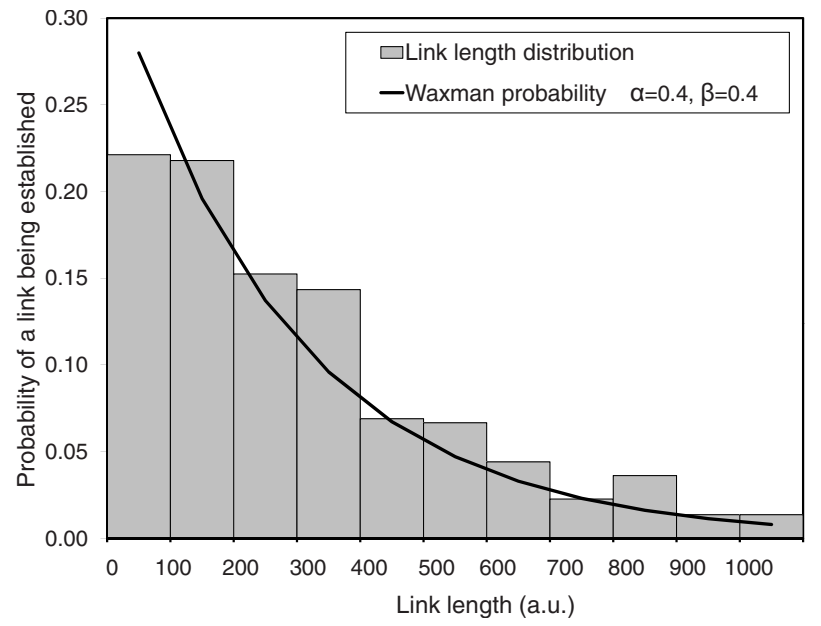

Fig. 7. Waxman link probability (6) with $\alpha=0.4$ and $\beta=0.4$ over a link length distribution. For the link length distribution we have considered 950 links of real-world networks presented in Table I.

same distribution of the respective real-world topologies at the 0.05 significance level. Referring to Fig. $8(a)$, we can see that the network topologies have $\delta_{\min } \geqslant 2$ and $\delta_{\max } \leqslant 8$ and $\langle\delta\rangle$ ranges from 2 to 4 with standard deviation in the range of 0.6 to 2.6. Considering all networks, we have $\langle\delta\rangle^{*}=2.8$. Figure 9 shows
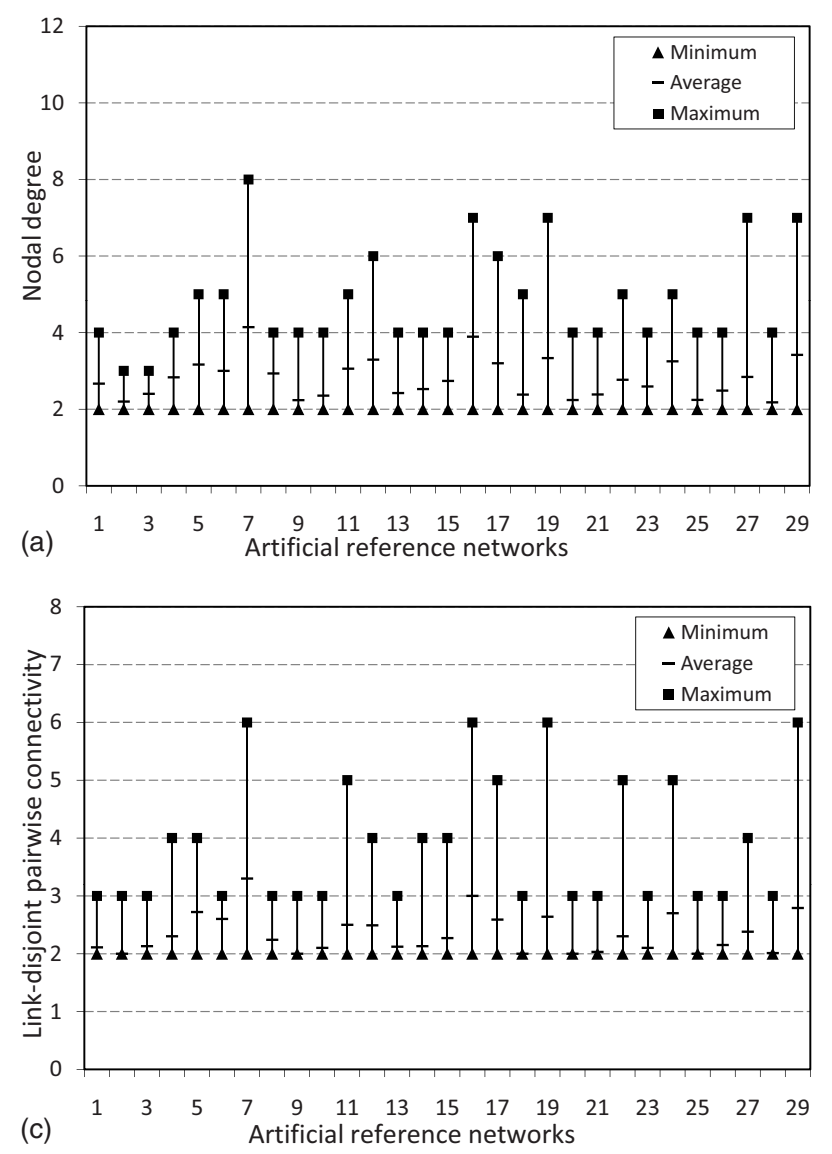

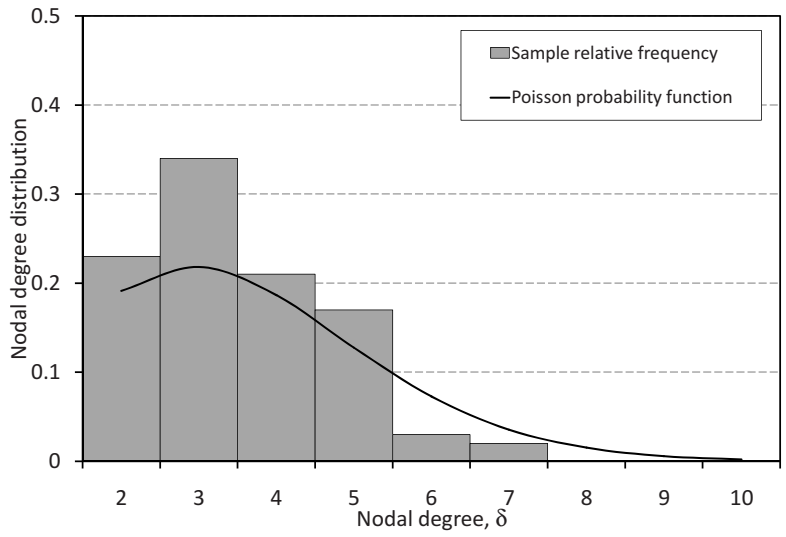

Fig. 9. Nodal degree relative frequency and the Poisson probability function with $\lambda=\langle\delta\rangle=3.42$ for a computer-generated topology with $N=100$ and $L=171$ (these values are identical to the USA 100 topology; see Fig. 2).

the nodal degree relative frequency and the Poisson probability function, with $\lambda=\langle\delta\rangle=3.42$, for a computergenerated topology with $N=100, L=171$ (these values correspond to those of the network presented in Fig. 2 ). As we can see, the degree distribution of the generated network tends to follow a Poisson probability function.
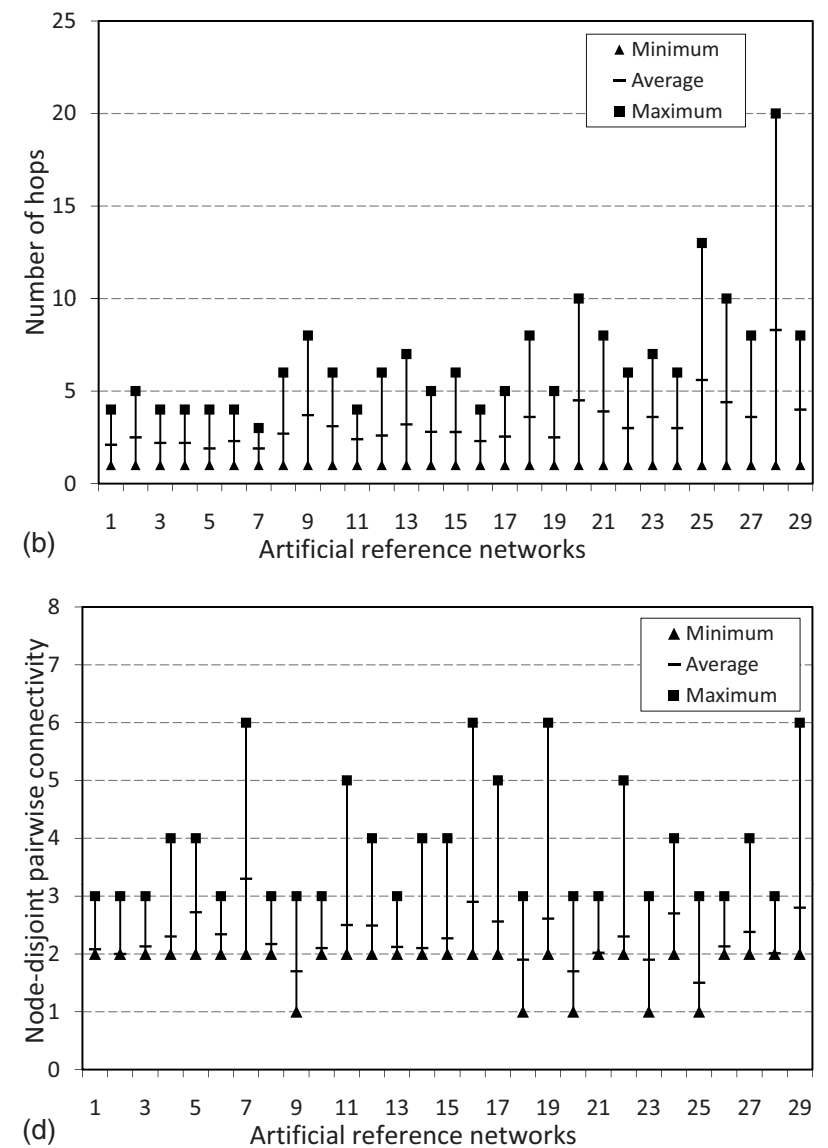

Fig. 8. The minimum, average, and maximum (a) nodal degree, (b) number of hops, (c) link-disjoint pairwise connectivity, and (d) nodedisjoint pairwise connectivity for the 29 computer-generated network topologies. 


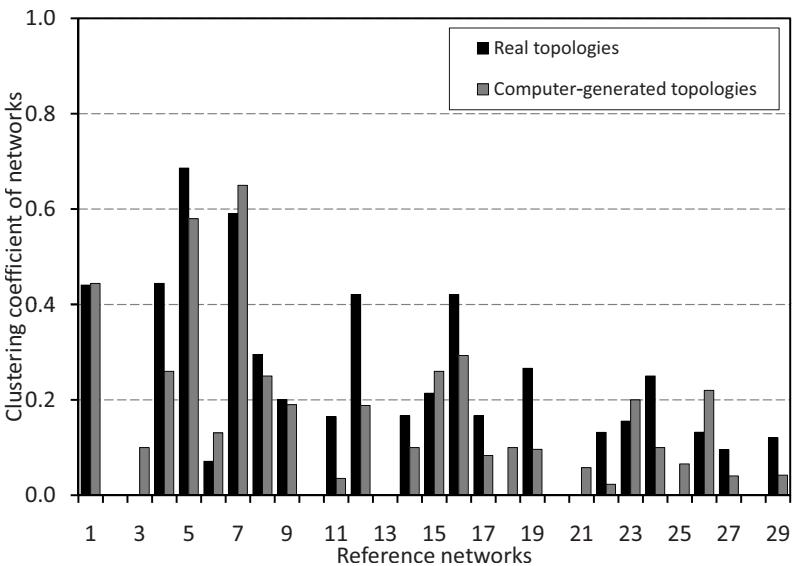

Fig. 10. Comparison between the clustering coefficient of real and computer-generated networks. The minimum and maximum deviation between each pair of networks (real and computer generated) is 0 and 0.23 , respectively. The average deviation is 0.07 .

Figure 8(b) shows that the number of hops ranges from 1 to 20 . The average number of hops, $\langle h\rangle$, ranges from 2 to 8.3 with a standard deviation in the range of 0.7-4.3. Considering all networks, we have $\langle h\rangle^{*}=3.2$.

In terms of both link- and node-disjoint pairwise connectivity, all the computer-generated topologies follow the same distribution of the respective realworld topologies at the 0.05 significance level. Figure 8(c) shows that all computer-generated network topologies have at least two link-disjoint paths between each pair of nodes, $\omega_{i j} \geqslant 2$, and at most six, $\omega_{i j} \leqslant 6$. The average link-disjoint pairwise connectivity, $\langle\Omega\rangle$, ranges from 2 to 3 independently of the network size and presents a standard deviation in the range of $0.1-1$. Considering all networks, we have $\langle\Omega\rangle^{*}=2.3$.

The node-disjoint pairwise connectivity satisfies 1 $\leqslant \theta_{i j} \leqslant 6$ [see Fig. 8(d)]. The average node-disjoint pairwise connectivity, $\langle\Theta\rangle$, for survivable topologies against single node failures ranges between 2 and 3 with a standard deviation in the range of $0-1.1$. Considering all networks, we have $\langle\Theta\rangle^{*}=2.26$.

In terms of the clustering coefficient of the network, all the computer-generated topologies follow the same distribution of the respective real-world topologies at

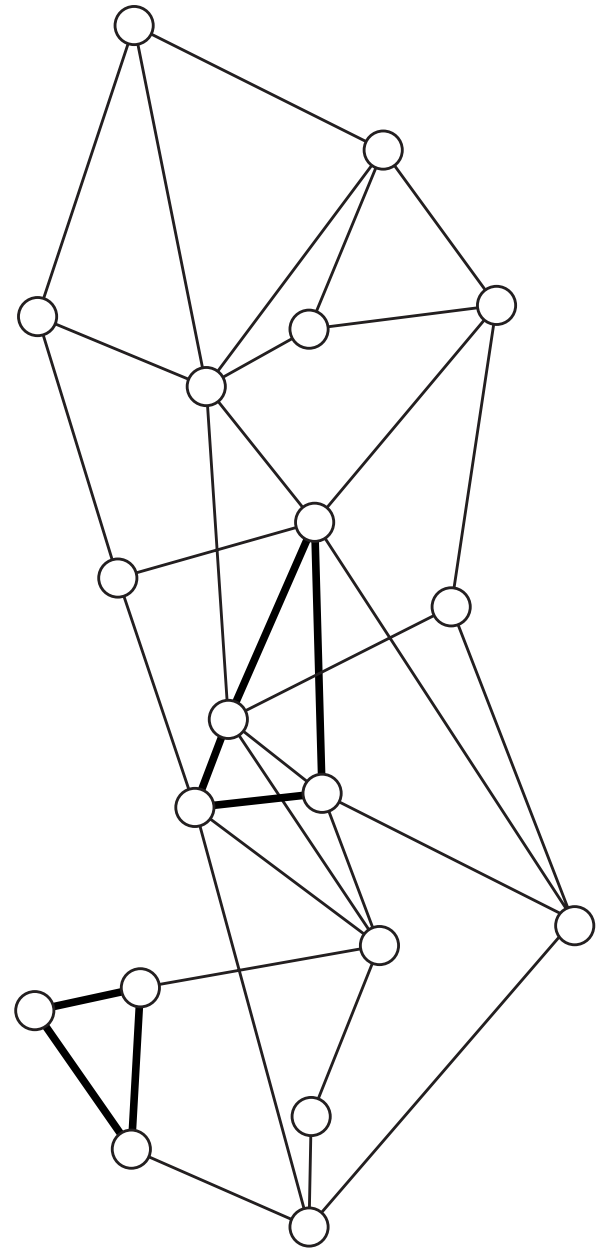

Fig. 11. Example of a computer-generated network topology for $N=19, L=37, A=100^{2}, R=12, d=2, \zeta=71, \alpha=0.4$, and $\beta=0.4$. Possible cycles inside regions are shown as highlighted links.

the 0.05 significance level. The clustering coefficient of nodes in our computer-generated networks ranges from 0 to 1 . The clustering coefficient of the networks ranges from 0 to 0.65 with standard deviation in the range of 0-0.4. Considering all networks, we have obtained $\langle c\rangle^{*}=0.16$. Figure 10 shows a comparison between the clustering coefficient of real and computergenerated networks. The average deviation is 0.07 .

In Table III we summarize the values of key vari-

TABLE III

Summary of the Values of Key Variables

\begin{tabular}{|c|c|c|c|c|c|c|c|c|}
\hline \multirow[b]{2}{*}{ Variable } & \multicolumn{2}{|c|}{ Minimum } & \multicolumn{2}{|c|}{ Average* } & \multicolumn{2}{|c|}{ Maximum } & \multicolumn{2}{|c|}{ Std. Deviation } \\
\hline & Real & CG & Real & CG & Real & $\mathrm{CG}$ & Real & CG \\
\hline$\delta$ & 2 & 2 & 2.80 & 2.80 & 10 & 8 & $0.4-2.0$ & $0.6-2.6$ \\
\hline$h$ & 1 & 1 & 3.40 & 3.20 & 21 & 20 & $0.7-4.6$ & $0.7-4.3$ \\
\hline$\omega$ & 2 & 2 & 2.25 & 2.30 & 7 & 6 & $0.0-0.9$ & $0.1-1.0$ \\
\hline$\theta$ & 1 & 1 & 2.21 & 2.26 & 7 & 6 & $0.0-1.0$ & $0.0-1.1$ \\
\hline$c$ & 0 & 0 & 0.19 & 0.16 & 1 & 1 & $0.0-0.4$ & $0.0-0.4$ \\
\hline
\end{tabular}


ables obtained from both real and computer-generated network topologies. We can see that, although a given number of nodes and links may produce a huge number of topologies, the computer-generated topologies effectively show statistics similar to the real-world optical transport networks. Figure 11 shows an example of a computer-generated network. The topology has the same number of nodes and links as the EON topology. The EON topology has $\delta$ varying from 1 to 7 with a mean of $3.89, h$ varying from 1 to 5 with a mean of 2.3 , $\omega$ from 2 to 7 with a mean of 2.9 , and $\theta$ from 2 to 6 with a mean of 2.6. The computergenerated topology has $\delta$ from 1 to 6 with a mean of $3.89, h$ varying from 1 to 5 with a mean of $2.4, \omega$ from 2 to 6 with a mean of 3 and $\theta$ from 2 to 6 with a mean of 2.8. Therefore, the statistics are similar to the ones presented in the EON network. Similar results were obtained with more than 50,000 computer-generated network topologies, when compared with the realworld network statistics.

\section{CONCLUSIONS}

We have studied the problem of generating realistic topologies for survivable transport networks. After identifying relevant characteristics of real-world transport networks, we proposed a method for generating survivable network topologies in which those characteristics have similar statistics to real-world networks. The proposed method is based on constraints that allow survivability and adequate nodal degree, number of hops, and link- and node-disjoint pairwise connectivity. We implemented and verified the proposed method by comparing the obtained results with practical networks. The values of the variables obtained from more than 50,000 computergenerated topologies are in agreement with those from the real-world reference networks, which validates the proposed method.

\section{ACKNOWLEDGMENTS}

This work was supported by the Fundação para Ciência e a Tecnologia-FCT, through the grant SFRH/BD/ 27545/2006, and PANORAMA project ADI/QREN3144 .

\section{REFERENCES}

[1] M. H. Gunes and K. Sarac, "Inferring subnets in router-level topology collection studies," in IMC '07: Proc. of the 7th ACM SIGCOMM Conf. on Internet Measurement, New York, NY, USA: ACM, 2007, pp. 203-208.

[2] M. Naldi, "Connectivity of Waxman topology models," Comput. Commun., vol. 29, no. 1, pp. 24-31, 2005.

[3] B. Waxman, "Routing of multipoint connections," IEEE J. Sel. Areas Commun., vol. 6, no. 9, pp. 1617-1622, Dec. 1988.
[4] M. Doar, "A better model for generating test networks," in Proc. of the IEEE Global Telecommunications Conf., GLOBECOM '96, Nov. 1996, pp. 86-93.

[5] E. Zegura, K. Calvert, and S. Bhattacharjee, "How to model an internetwork," in Proc. of the 15th Annu. Joint Conf. of the IEEE Computer Societies. Networking the Next Generation. INFOCOM '96, vol. 2, Mar. 1996, pp. 594-602.

[6] L. Cheng, N. Hutchinson, and M. Ito, "Realnet: a topology generator based on real Internet topology," in Proc. of the 22nd Int. Conf. on Advanced Information Networking and Applications, AINAW '08, Mar. 2008, pp. 526-532.

[7] M. Faloutsos, P. Faloutsos, and C. Faloutsos, "On power-law relationships of the Internet topology," in Proc. of the Conf. on Applications, Technologies, Architectures, and Protocols for Computer Communication, SIGCOMM '99, New York, NY, USA: ACM, 1999, pp. 251-262.

[8] C. Jin, C. J. Qian, and S. Jamin, "Inet: Internet topology generator," Technical Report CSE-TR-433-00, EECS Department, University of Michigan, 2000.

[9] D. Magoni, "Nem: a software for network topology analysis and modeling," in Proc. of the 10th IEEE Int. Symp. on Modeling, Analysis and Simulation of Computer and Telecommunications Systems, MASCOTS '02, Oct. 2002, pp. 364-371.

[10] C. Palmer and J. Steffan, "Generating network topologies that obey power laws," in Proc. of the IEEE Global Telecommunications Conf., GLOBECOM '00, vol. 1, Nov. 2000, pp. 434-438.

[11] A. Medina, A. Lakhina, I. Matta, and J. Byers, "Brite: an approach to universal topology generation," in Proc. of the 9th Int. Symp. on Modeling, Analysis and Simulation of Computer and Telecommunication Systems, MASCOTS '01, Aug. 2001, pp. $346-353$.

[12] A.-L. Barabasi and E. Bonabeau, "Scale-free networks," Sci. Am., vol. 288, no. 5, pp. 50-59, May 2003.

[13] VIA Networks, http://www.vianetworks.net/datacenterglobalnetwork.php.

[14] Bulgarian Research and Education Network-BREN, BREN_map_2008_EN.png, http://www.bren.acad.bg/images.

[15] Rede Nacional de Pesquisa-RNP, http://www.rnp.br/en/ backbone/index.php.

[16] The very-high-performance Backbone Network ServicevBNS, http://www.stanford.edu/services/internet2/vbns.html.

[17] Czech Education and Scientific NETwork-CESNET, http:// www.cesnet.cz/provoz/zatizeni.

[18] National Science Foundation Network-NSFNET, http:// www.ucpr.edu.co/paginas/revista57/auge.htm.

[19] D. Colle, S. De Maesschalck, C. Develder, P. Van Heuven, A. Groebbens, J. Cheyns, I. Lievens, M. Pickavet, P. Lagasse, and P. Demeester, "Data-centric optical networks and their survivability," IEEE J. Sel. Areas Commun., vol. 20, no. 1, pp. 6-20, Jan. 2002.

[20] Austrian Academic Computer Network-ACOnet, http:// www.aco.net/technologie.html.

[21] Mzima Backbone Network, network_map_rev0001b.swf, http:// www.mzima.net/gfx.

[22] Slovenia Academic and Research Network-ARNES, http:// www.arnes.si/backbone.htm.

[23] C. T. Politi, H. Haunstein, D. A. Schupke, S. Duhovnikov, G. Lehmann, A. Stavdas, M. Gunkel, J. Martensson, and A. Lord, "Integrated design and operation of a transparent optical network: a systematic approach to include physical layer awareness and cost function," IEEE Commun. Mag., vol. 45, no. 2, pp. 40-47, Feb. 2007.

[24] RedIRIS Network, http://www.rediris.es/red.

[25] National LambdaRail, http://noc.nlr.net.

[26] Memorex Network, http://www.memorex-telex.cz/ products02.php.

[27] Canada's Advanced Network-CANARIE, http:// www.canarie.ca/canet4/connected/map.html. 
[28] M. Klinkowski, F. Herrero, D. Careglio, and J. Sol-Pareta, "Adaptive routing algorithms for optical packet switching networks," in Proc. of the Conf. on Optical Network Design and Modelling, ONMD '05, Feb 2005, pp. 235-241.

[29] Advanced Research Projects Agency Network-ARPANET, http://www.optical-network.com/topology.php.

[30] Polish Optical Internet-PIONIER, http://www.pionier.gov.pl/ network/index.htm.

[31] Cox High Speed Internet Backbone, mapNationalInt.gif, http:// www.coxbusiness.com/value/lalaunch/images.

[32] Slovak Academic Network-SANET, http://www.sanet.sk/en/ siet_topologia.shtm.

[33] Internet's New Backbone Network, http://www.internet2.edu/ network/.

[34] J. Pedro, A. Teixeira, P. Monteiro, and J. Pires, "On a Portuguese backbone network of reference," in Proc. of the Symp. on Enabling Optical Networks and Sensors, SEON'05, June 2005, pp. 80-84.

[35] National Telecommunication Network for Technology, Education and Research-RENATER, http://www.renater.fr/ spip.php?rubrique12\&lang $=$ en .

[36] GEANT Backbone Topology-GEANT2, http:// www.geant2.net/server/show/nav.00d007009.

[37] Louisiana Optical Network Initiative-LONI, http:// www.loni.org/images/LONI_Architecture_060106.jpg.

[38] Metrona Network, http://www.metronap.net/NetworkMap.aspx

[39] Omnicom Telecommunications Network Map, http:// www.omnicom-telecom.com/company.html.

[40] Internet2 Network, http://www.internet2.edu/network.

[41] S. K. Korotky, "Network global expectation model: a statistical formalism for quickly quantifying network needs and costs," $J$. Lightwave Technol., vol. 22, no. 3, pp. 703-722, Mar. 2004.

[42] J. H. Stapleton, Models for Probability and Statistical Inference: Theory and Applications. Hoboken, NJ, USA: WileyInterscience, 2007.

[43] A.-L. Barabasi, "The architecture of complexity," IEEE Control Syst., vol. 27, no. 4, pp. 33-42, Aug. 2007.

[44] J.-P. Vasseur, M. Pickavet, and P. Demeester, Network Recovery: Protection and Restoration of Optical, SONET-SDH, IP, and MPLS. San Francisco, CA, USA: Morgan Kaufmann, 2004.

[45] W. D. Grover, Mesh-Based Survivable Networks: Options and Strategies for Optical, MPLS, SONET, and ATM Networking. NJ: Prentice Hall, 2004.

[46] S. Ramamurthy, L. Sahasrabuddhe, and B. Mukherjee, "Survivable WDM mesh networks," J. Lightwave Technol., vol. 21, no. 4, pp. 870-883, Apr. 2003.

[47] N. Takahashi, "On clustering coefficients of graphs with the fixed numbers of vertices and edges," in European Conf. on Circuit Theory and Design, 2009. ECCTD 2009, Aug. 2009, pp. 814-817.

[48] G. Siganos, M. Faloutsos, P. Faloutsos, and C. Faloutsos, "Power laws and the as-level Internet topology," IEEE/ACM Trans. Netw., vol. 11, no. 4, pp. 514-524, Aug. 2003.

[49] H. Tangmunarunkit, R. Govindan, S. Jamin, S. Shenker, and W. Willinger, "Network topology generators: degree-based vs. structural," in Proc. of the Conf. on Applications, Technologies, Architectures, and Protocols for Computer Communication, SIGCOMM '02, vol. 32, no. 4, New York, NY, USA: ACM, Aug. 2002, pp. 147-159.

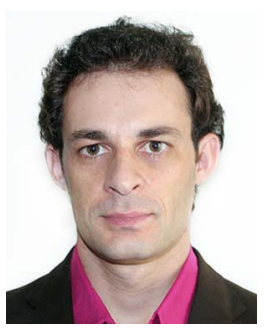

Claunir Pavan received a degree in informatics technology from the West University of Santa Catarina-UNOESC, SC, Brazil, in 2000 and an M.Sc. degree in computer science from the Federal University of Santa Catarina, SC, Brazil, in 2003. From 2003 to 2005 he was with the UNOESC, where he taught courses on information systems and was head of the CTICtechnology information and communications department. In 2005, he moved to Portugal to work as a researcher with the Integration Strategies for IP over WDM Networks project at the Institute of Telecommunications. He is currently working toward his Ph.D. degree at the Department of Electronics, Telecommunications and Informatics, University of Aveiro, Portugal. His main research interests include the dimensioning of optical multilayer networks.

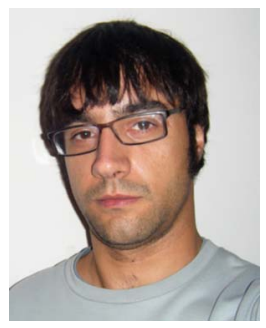

Rui Manuel Morais was born in Gouveia, Portugal. He received a degree in applied mathematics and computation and an M.Sc. degree in mathematics and applications, both from Universidade de Aveiro, Aveiro, Portugal, in 2006 and 2008, respectively. He joined Instituto de Telecomunicações in 2007 working on the dimensioning of optical networks. His main research interests are the dimensioning problem with incomplete information and the optical network design

problem.

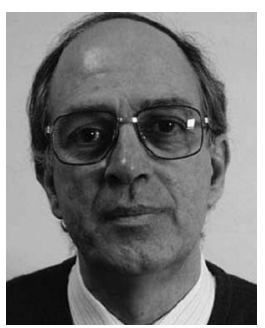

José R. Ferreira da Rocha was born in Mozambique. He received an M.Sc. degree in telecommunication systems and a Ph.D. degree in electrical engineering, both from the University of Essex, Essex, UK, in 1980 and 1983 , respectively. He participates actively in the Telecommunications Institute, a national R\&D nonprofit organization, where he is a member of the Management Committee (Aveiro branch) and the $\mathrm{Na}$ tional Coordinator for the Optical Communications Area. He has coordinated the University of Aveiro, Aveiro, Portugal, and Telecommunications Institute participation in various projects included in the following European Union (EU) R\&D Programs in the area of telecommunications: RACE, RACE II, ACTS, and IST. In the past few years, he has acted as a Technical Auditor, Evaluator, and Independent Observer for the evaluation of projects submitted to various EU R\&D Programs. He has also participated in various project evaluation boards set up by the Engineering and Physical Sciences Research Council (EPSRC), UK. By invitation of the ACTS Management Committee, he participated in the Expert Groups on Visionary Research in Communications, aiming to create a bridge between the activities carried out in the Fourth and the Fifth Framework Programs on EU activities in the field of research, technological development, and demonstration. $\mathrm{He}$ is currently a Full Professor at the University of Aveiro. He has published about 170 papers, mainly in international journals and conferences. His present research interests include modulation formats and receiver design for very high capacity (above $40 \mathrm{~Gb} / \mathrm{s}$ ) optical communication systems based on linear and nonlinear transmission and wavelength division multiplexing (WDM) optical networks. 


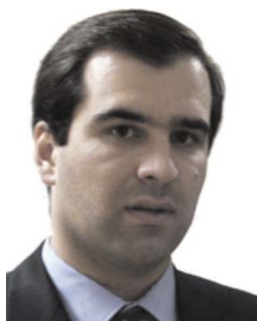

Armando Nolasco Pinto was born in Oliveira do Bairro, Portugal, in 1971. He graduated in electronic and telecommunications engineering in 1994, and he obtained a Ph.D. degree in electrical engineering in 1999, both from the University of Aveiro, Aveiro, Portugal. In 1995 and 1996, as part of his Ph.D. studies, he worked with Professor Govind Agrawal at the Institute of Optics, University of Rochester, Rochester, NY, USA. In 2000, he became an Assistant Professor at the Electrical, Telecommunications and Informatics De- partment, University of Aveiro, and a Researcher at the Institute of Telecommunications, Aveiro. During the academic year of 20062007 he was a Visiting Professor at the Institute of Optics, University of Rochester, Rochester, NY, USA. At present, he leads a research group at the Institute of Telecommunications focused on high-speed optical communication systems and networks. He has published more than 100 scientific papers in international journals and conferences. Dr. Pinto is a member of the Optical Society of America (OSA) and a senior member of the Institute of Electrical and Electronics Engineers (IEEE). 